\title{
MHC class II pathway mediates adipose inflammation
}

Obesity is associated with increased MHC class II antigen presentation in adipocytes, which leads to increased proinflammatory T-cell activity in adipose tissue. These findings, from Willa Hsueh's laboratory (Houston, USA), are a new step in clarifying the link between obesity and inflammation.

"Chronic adipose tissue inflammation is a hallmark of obesity, leading to obesity-induced insulin resistance," comments study researcher Tuo Deng. The proinflammatory activity of T cells is known to be increased in adipose tissue of humans and mice. "These $\mathrm{T}$ cells release IFN- $\gamma$ to induce macrophage activation," Deng explains. "However, the critical signal that induces adipose tissue T-cell activation is unknown."

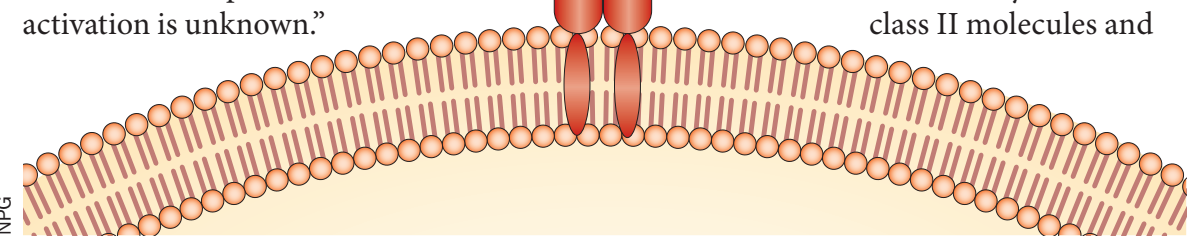

Deng and colleagues performed microarray analysis in adipocytes isolated from biopsies of 44 women with obesity and saw that expression of genes involved in MHC class II antigen presentation and processing was increased. Expression of genes of the MHC class II pathway

was also upregulated in mouse adipocytes after 2 weeks of a high-fat diet, when compared with expression of these genes in chow-fed mice. These changes occurred before macrophage accumulation in adipose tissue, which was only detected 4 weeks after the beginning of the diet. Further experiments in mouse cells showed that activation of $\mathrm{T}$ cells in adipocytes

MHC class II is mediated by MHC class II molecules and is antigen-dependent. Finally, the researchers saw that MHC class II knockout mice fed a high-fat diet have less inflammation and insulin resistance than wild-type mice fed the same diet.

"Our study suggests that adipocytes work in conjunction with macrophages, 'professional' antigen-presenting cells, to send messages to $\mathrm{T}$ cells that induce inflammation during excess caloric intake," says Deng.

The researchers are now aiming to identify the specific antigens responsible for the activation of T cells by MHC class II molecules in adipocytes. "We would have a new highly-specific approach to target inflammation in patients with obesity," Deng concludes.

Joana Osório

Original article Deng, T. et al. Class II major histocompatibility complex plays an essential role in obesity-induced adipose inflammation. Cell Metab. doi:10/1016/j.cmet.2013.02.009 